\title{
In vivo photoacoustic and ultrasonic mapping of rat sentinel lymph nodes with a modified commercial ultrasound imaging system
}

\begin{abstract}
Todd N. Erpelding, Chulhong Kim, Manojit Pramanik, Zijian Guo, John Dean, et al.
\end{abstract}

Todd N. Erpelding, Chulhong Kim, Manojit Pramanik, Zijian Guo, John Dean, Ladislav Jankovic, Konstantin Maslov, Lihong V. Wang, "In vivo photoacoustic and ultrasonic mapping of rat sentinel lymph nodes with a modified commercial ultrasound imaging system," Proc. SPIE 7564, Photons Plus Ultrasound: Imaging and Sensing 2010, 756418 (23 February 2010); doi: 10.1117/12.841858

SPIE. Event: SPIE BiOS, 2010, San Francisco, California, United States 


\title{
In vivo photoacoustic and ultrasonic mapping of rat sentinel lymph nodes with a modified commercial ultrasound imaging system
}

Todd N. Erpelding*a, Chulhong Kim ${ }^{\mathrm{b}}$, Manojit Pramanik ${ }^{\mathrm{b}}$, Zijian Guo ${ }^{\mathrm{b}}$, John Dean ${ }^{\mathrm{a}}$, Ladislav Jankovic $^{\mathrm{a}}$, Konstantin Maslov ${ }^{\mathrm{b}}$, and Lihong V. Wang ${ }^{\mathrm{b}}$

${ }^{a}$ Philips Research North America, 345 Scarborough Rd., Briarcliff Manor, NY, USA 10510; boptical Imaging Laboratory, Department of Biomedical Engineering, Washington University in St. Louis, 1 Brookings Dr., St. Louis, MO, USA 63130

\begin{abstract}
Sentinel lymph node biopsy (SLNB) has become the standard method for axillary staging in breast cancer patients, relying on invasive identification of sentinel lymph nodes (SLNs) following injection of blue dye and radioactive tracers. While SLNB achieves a low false negative rate (5-10\%), it is an invasive procedure requiring ionizing radiation. As an alternative to SLNB, ultrasound-guided fine needle aspiration biopsy has been tested clinically. However, ultrasound alone is unable to accurately identify which lymph nodes are sentinel. Therefore, a non-ionizing and noninvasive detection method for accurate SLN mapping is needed.

In this study, we successfully imaged methylene blue dye accumulation in vivo in rat axillary lymph nodes using a Phillips iU22 ultrasound imaging system adapted for photoacoustic imaging with an Nd:YAG pumped, tunable dye laser. Photoacoustic images of rat SLNs clearly identify methylene blue dye accumulation within minutes following intradermal dye injection and co-registered photoacoustic/ultrasound images illustrate lymph node position relative to surrounding anatomy. To investigate clinical translation, the imaging depth was extended up to $2.5 \mathrm{~cm}$ by adding chicken breast tissue on top of the rat skin surface. These results raise confidence that photoacoustic imaging can be used clinically for accurate, noninvasive SLN mapping.
\end{abstract}

Keywords: Photoacoustic imaging, ultrasound imaging, sentinel lymph node, breast cancer

\section{INTRODUCTION}

Sentinel lymph node biopsy (SLNB) has emerged as the preferred method for axillary lymph node staging of clinically node-negative breast cancer. ${ }^{1-3}$ The first lymph nodes to receive drainage from the primary tumor are defined as sentinel and are most likely to be positive for metastases. Axillary lymph node staging in clinically node-negative breast cancer patients often proceeds in two surgical stages. If the pathological SLNB results are positive, then the patient undergoes a second more involved surgery, axillary lymph node dissection (ALND) to remove all level I and II lymph nodes. Typically, 10-30 lymph nodes are removed during ALND, which is associated with nerve injury, lymphedema, and decreased range of motion in the shoulder. If the SLNB results are negative, then full axillary lymph node dissection (ALND) can be avoided. SLNB following injection of radioactive tracers and blue dye achieves high identification rates $(90-97 \%)$ and low false-negative rates $(5-10 \%){ }^{4-6}$

SLNB has revolutionized the assessment of the axilla for patients newly diagnosed with breast cancer. However, SLNB is a time-consuming procedure, requiring a considerable amount of operating room time to harvest the nodes and significant time for the histological diagnosis. Furthermore, while SLNB reduces the morbidity and side-effects as compared to ALND, it still remains an invasive surgical procedure with potential post-operative complications including lymphedema, seroma formation, sensory nerve injury and range of motion limitations. ${ }^{7,8}$ A recent clinical trial found that $74.2 \%$ (3989 of 5379) of breast cancer patients who underwent sentinel lymph node (SLN) resection were pathologically negative. ${ }^{9}$ For the majority of SLNB patients, a preoperative and noninvasive alternative could more efficiently stage the axilla without requiring surgical intervention. Noninvasive identification of SLNs would allow percutaneous needle biopsies to sample the lymph nodes that are most likely to contain metastatic tumor cells.

*Email: todd.erpelding@philips.com

Photons Plus Ultrasound: Imaging and Sensing 2010, edited by Alexander A. Oraevsky, Lihong V. Wang, Proc. of SPIE Vol. 7564, 756418 · (c) 2010 SPIE · CCC code: 1605-7422/10/\$18 · doi: 10.1117/12.841858 
Photoacoustic imaging is a non-ionizing and noninvasive hybrid imaging technique that can supply strong optical absorption contrasts with high ultrasonic spatial resolution. ${ }^{10,11}$ Photoacoustic imaging relies on the photoacoustic effect for generating pressure waves under thermal and stress confinement conditions. A short pulsed laser is used to irradiate tissue causing optical absorption, rapid thermoelastic expansion, and acoustic pressure wave generation. Photoacoustic pressure waves can be detected using ultrasound array transducers for efficient parallel data acquisition. A table-top photoacoustic imaging system based on mechanical raster scanning has been applied to identify rat SLNs in vivo using methylene blue, gold nanoparticles, and single-walled carbon nanotubes. ${ }^{12-15}$ However, the imaging speed of this system $\left(\sim 18 \mathrm{~min}\right.$ for $24 \times 24 \mathrm{~mm}^{2}$ field of view) limits its clinical applicability due to mechanical scanning.

In this study, we report the development of a combined photoacoustic and ultrasound imaging system ${ }^{16}$ that can potentially provide accurate, non-ionizing, and noninvasive mapping of human SLNs to guide fine needle aspiration biopsies (FNAB) for axillary staging in breast cancer patients.

\section{METHODOLOGY}

\subsection{Animal and dye preparation}

Animal protocols were approved by the Washington University Animal Studies Committee and animal handling was performed according to the guidelines on the care and use of laboratory animals. Healthy Sprague Dawley rats were initially anesthetized using a mixture of ketamine $(85 \mathrm{mg} / \mathrm{kg})$ and xylazine $(15 \mathrm{mg} / \mathrm{kg})$. The hair in the left axillary region was gently depilated before imaging. Photoacoustic and ultrasound images were collected before and after $0.1 \mathrm{~mL}$ intradermal injection of $1 \%$ methylene blue dye $(10 \mathrm{mg} / \mathrm{mL}$, American Reget, Inc., Shirley, NY) into the left forepaw pad. Animals stayed under full anesthesia throughout the experiment using isofluorane gas $(1 \mathrm{~L} / \mathrm{min}$ oxygen and $0.75 \%$ isofluorane, Euthanex Corp., Palmer, PA). Simultaneously, both heart rate and saturation of peripheral oxygen (SPO2) were monitored using a pulse oximeter $(8600 \mathrm{~V}$, Nonin Medical, Inc., Plymouth, MN). After imaging, animals were euthanized with an overdose of pentobarbital, and SLNs were dissected.

\subsection{Photoacoustic imaging system}

A one of a kind photoacoustic system (Figure 1) has been built around a Philips iU22 ultrasound imaging system (Philips Healthcare, Andover, MA).$^{17}$ The modified channel board architecture allowed access to raw per-channel photoacoustic data, while retaining all imaging capabilities of an actual commercial ultrasound scanner. Raw photoacoustic and ultrasound data were transferred to a custom-built data acquisition system for image display and post-processing, providing dual-modality imaging capability. Photoacoustic images were reconstructed using a Fourier beamforming algorithm ${ }^{18}$ implemented in Matlab (MathWorks Inc., Natick, MA), generating a photoacoustic B-mode image from one laser shot. An FPGA-based electronic board synchronized iU22 data acquisition with the laser firing. For this study, the linear array L8-4 probe with nominal bandwidth 4-8 MHz was used.

Light from a tunable dye laser (ND6000, Continuum, Santa Clara, CA), pumped by a Q-switched Nd:YAG laser (Brilliant B, Quantel, Les Ulis, France) with 6.5-ns pulse duration and 10-Hz pulse repetition rate, was delivered through a high temperature epoxy, multimode fiber bundle (SCHOTT Fiber Optics, Southbridge, MA). Photoacoustic images were acquired using $658 \mathrm{~nm}$ light to produce the strongest photoacoustic signals from methylene blue based on the laser power spectrum. The light fluence on the skin was less than $5 \mathrm{~mJ} / \mathrm{cm}^{2}$, well within American National Standards Institue (ANSI) safety limits. ${ }^{19}$

The fiber bundle was physically attached to an ultrasound probe to enable dual-modality photoacoustic and ultrasonic imaging. The final optical illumination pattern on the skin surface was rectangle-shaped $(3 \mathrm{~cm} \times 1 \mathrm{~cm}$ along the $\mathrm{X}$ and $\mathrm{Y}$ axes, respectively). The imaging plane of the ultrasound probe was coaxially aligned with the oblique incidence $\left(\sim 45^{\circ}\right)$ of the rectangle-shaped optical beam on the targeted area. The photoacoustic probe was partially immersed in a water tank which had an opening at the bottom sealed with a thin, disposable, clear membrane. Rats were placed below the water tank, in contact with the membrane via ultrasonic coupling gel. 


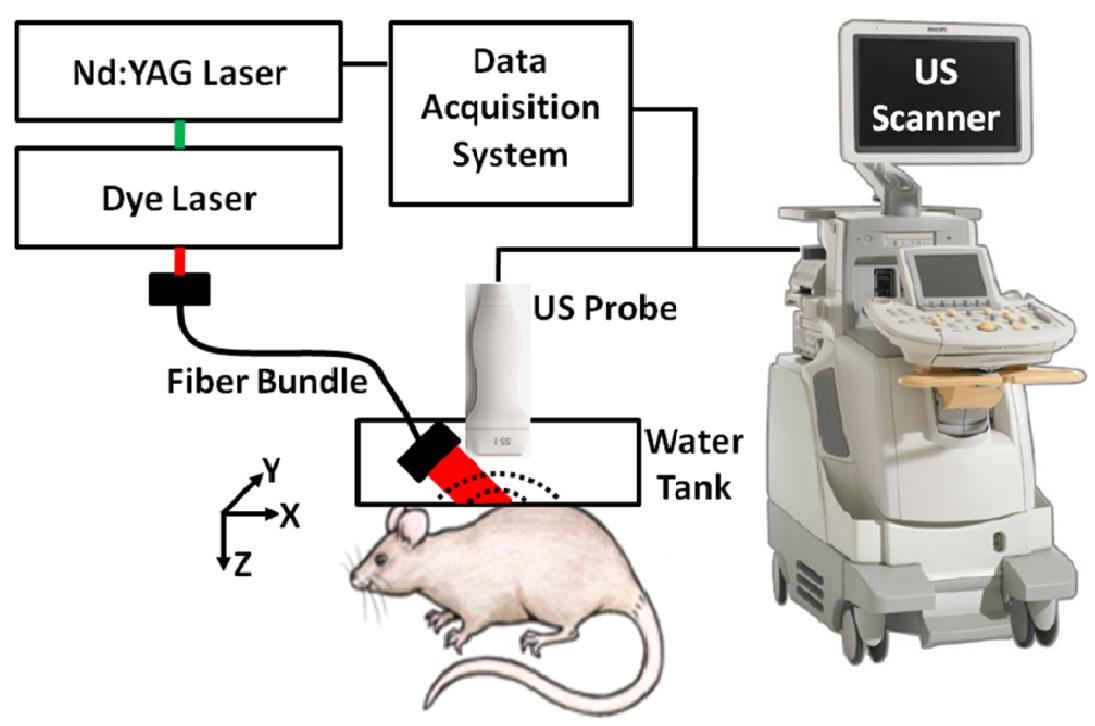

Figure 1. Experimental setup of photoacoustic imaging system adapted from a modified Philips iU22 ultrasound (US) imaging system (Philips Healthcare, Andover, MA).

\section{RESULTS}

\subsection{Phantom imaging}

Photoacoustic and ultrasound images of a suture wire phantom target using the modified iU22 system (L8-4 probe) are given in Figure 2. The phantom consisted of 3 suture wires $(\sim 0.5 \mathrm{~mm}$ thick) positioned along the long axis of the transducer. The bottom 2 wires were crisscrossed to form an " $X$ ". The co-registered photoacoustic/ultrasound image (Figure 2c) gives a qualitative demonstration of the axial resolution and good spatial agreement that ensures proper synchronization between laser firings and data collection. The hyperechoic regions in the ultrasound image above the suture wires are from the phantom holder, which is made of transparent plexiglass and does not generate a photoacoustic signal.
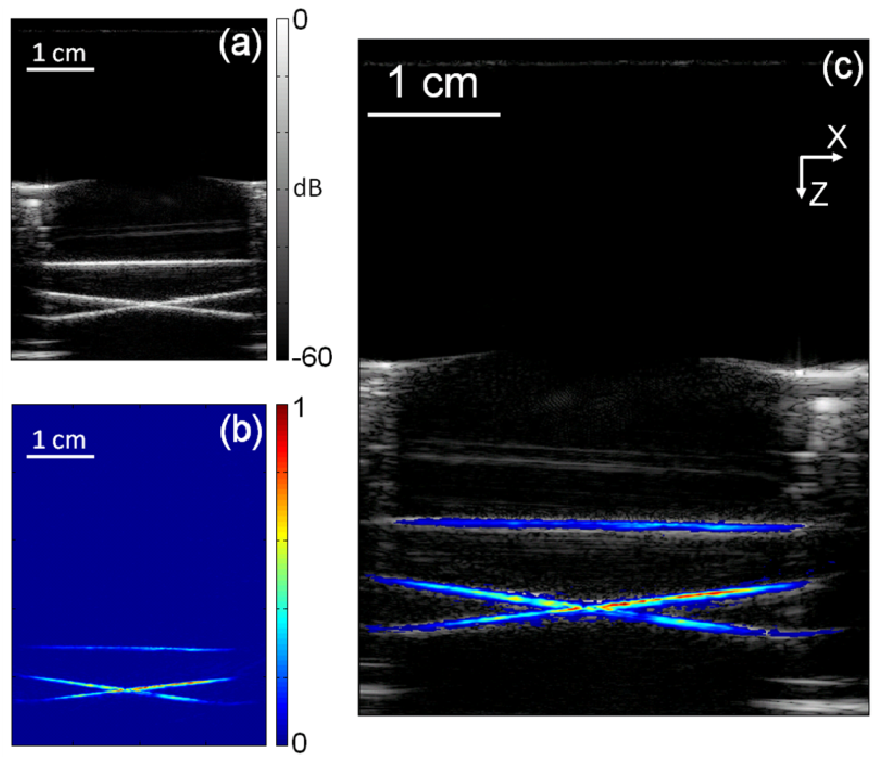

Figure 2. (a) Ultrasound image of suture wire phantom. (b) Photoacoustic image of suture wire phantom. (c) Coregistered photoacoustic/ultrasound image of suture wire phantom. 


\subsection{In vivo rat SLN imaging}

Photoacoustic images of the left axillary region in rats were acquired before and after methylene blue injection (Figure 3) to investigate the feasibility of photoacoustic and ultrasonic SLN mapping. Superficial blood vessels and the skin surface can be detected in the pre-injection photoacoustic B-mode image (Figure 3a). Soon after injection, methylene blue accumulates in SLNs, as detected photoacoustically. Figure $3 \mathrm{~b}$ shows a photoacoustic B-mode image from the SLN acquired 20 min following methylene blue injection. Pre- and post-injection images are displayed with the same dynamic range for appreciation of the strong photoacoustic signal from SLNs following methylene blue accumulation. Postinjection photoacoustic images are overlaid on structural ultrasound B-mode images in Figure 3c.

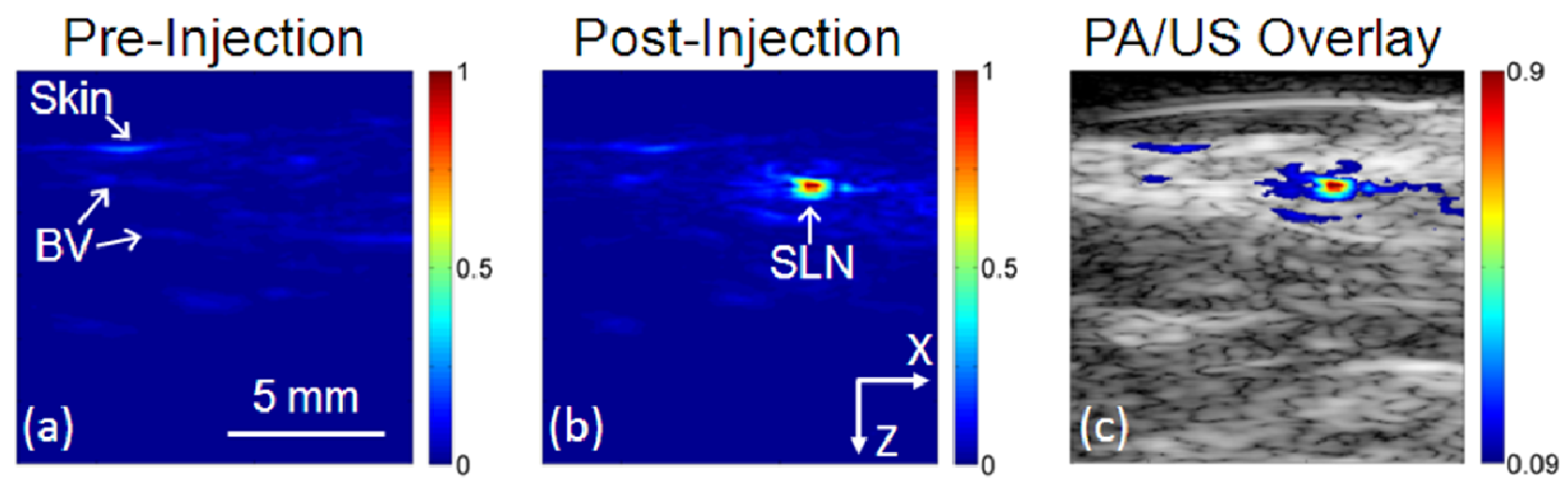

Figure 3. Noninvasive photoacoustic (PA) B-mode images of a rat sentinel lymph nodes (SLNs) acquired in vivo with the L8-4 probe. (a) Control photoacoustic image acquired before methylene blue injection showing the skin surface and superficial blood vessels (BVs). (b) Photoacoustic images acquired 20 minutes following methylene blue injection. (c) Co-registered photoacoustic and ultrasound (US) images acquired 20 min following methylene blue injection.

A sample photograph of the rat axillary region imaged during experiments is shown in Figure 4a. A post-mortem photograph of the same rat after the experiment was completed is shown in Figure 4b, with the skin removed. The methylene blue dyed SLN was visually identified in the left axilla. Lymph nodes were typically $3 \mathrm{~mm}$ by $2 \mathrm{~mm}$ in size, consistent with the size obtained from photoacoustic images.
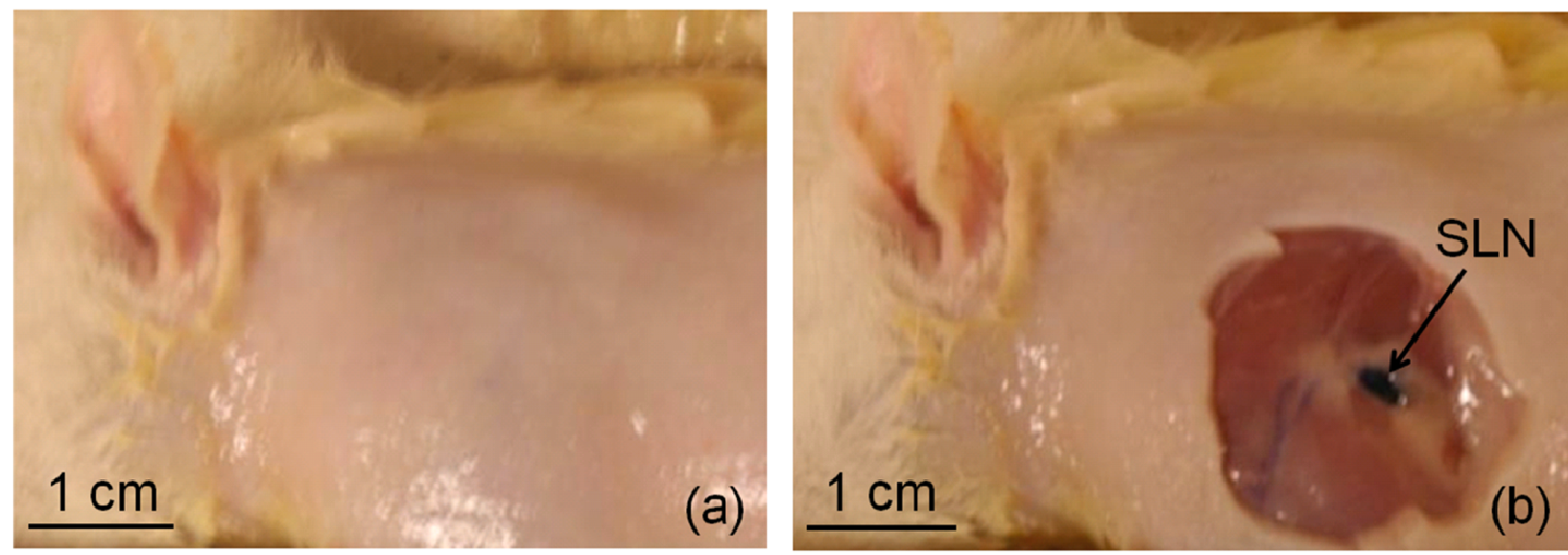

Figure 4. (a) Photograph of rat as prepared for photoacoustic imaging. (b) Post-mortem photograph of rat acquired after photoacoustic imaging. Note the skin was removed after photoacoustic imaging was completed.

The imaging depth for rat lymph nodes was increased by adding chicken breast tissue on top of the rat skin surface. Figure 5 demonstrates the ability to image a SLN located $2.5 \mathrm{~cm}$ below the top surface of the overlying chicken breast using the L8-4 probe. No signal averaging was needed at these depths. 


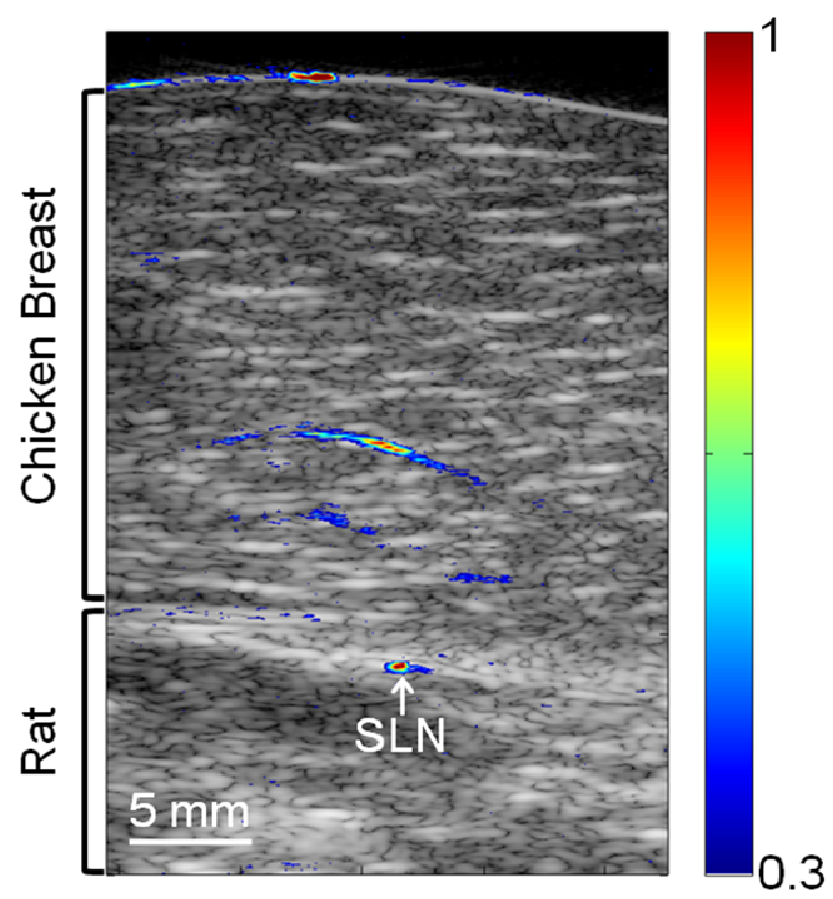

Figure 5. Co-registered photoacoustic (PA) and ultrasound B-mode images of rat sentinel lymph nodes (SLNs) acquired in vivo with added biological tissue for increased imaging depth. (a) SLN image acquired with the L8-4 probe through an additional 2-cm chicken breast 12 min following methylene blue injection.

\section{DISCUSSION}

Noninvasive photoacoustic and ultrasound imaging has been used to map rat SLNs following methylene blue injection. The method is highly translatable since it demonstrates photoacoustic detection of SLNs using a modified clinical ultrasound imaging system and methylene blue is routinely used during SLNB. Two ultrasound probes successfully imaged uptake of methylene blue in SLNs and enable probe selection based on imaging requirements (i.e. depth, sensitivity, and resolution).

In most cases, rat axillary lymph nodes are only a few millimeters below the skin surface. Human axillary lymph nodes are deeper but larger. Photoacoustic SLN mapping was demonstrated at an imaging depth of $2.5 \mathrm{~cm}$, without any signal averaging, by adding chicken breast tissue on top of the rat axillary skin. In the current study, optical fluence on the skin was less than $5 \mathrm{~mJ} / \mathrm{cm}^{2}$, which can be increased by a factor of four while still conforming to the maximal permissible exposure allowed by ANSI limits $\left(20 \mathrm{~mJ} / \mathrm{cm}^{2}\right) .{ }^{19}$ Since photoacoustic signal strength depends on optical fluence and absorption, stronger optical fluence will enable photoacoustic SLN detection at even greater depths. Based on these results, photoacoustic imaging should be able to noninvasively detect axillary SLNs at most depths encountered in humans.

Successful noninvasive photoacoustic mapping of rat SLNs through $\sim 2.5 \mathrm{~cm}$ of tissue motivates further evaluation of this technique and imaging devices in future human trials. A noninvasive method for accurately mapping axillary SLNs in breast cancer patients could be used to guide FNAB. In some patients, axillary ultrasound imaging can identify abnormal-appearing lymph nodes based on morphological features. Needle biopsy can be performed in these instances but only a positive result is informative. Negative needle biopsies do not rule out the presence of malignancy. The ability to noninvasively identify SLNs would enable needle biopsies to target lymph nodes most likely to contain metastatic tumors. Combined photoacoustic and ultrasound-guided FNAB of SLNs potentially could eliminate invasive axillary staging procedures.

In summary, noninvasive photoacoustic and ultrasonic mapping of rat SLNs and lymphatic vessels has been successfully demonstrated using methylene blue dye with a modified commercial ultrasound imaging system. 


\section{ACKNOWLEDGEMENTS}

This research was supported by National Institutes of Health grants U54 CA136398 (Network for Translational Research), R01 EB000712, R01 NS46214, and R01 EB008085. We thank Victor Gornstein and Adam Schleicher for their contributions to modifying the Philips iU22 ultrasound imaging system. We thank Julie Margenthaler for helpful discussions on clinical breast cancer staging and treatment. LVW has a financial interest in Microphotoacoustics, Inc. and Endra, Inc., which, however, did not support this work. TNE, JD, and LJ are employees of Philips Research.

\section{REFERENCES}

[1] Krag, D. N., Weaver, D. L., Alex, J. C., and Fairbank, J. T., "Surgical resection and radiolocalization of the sentinel lymph node in breast cancer using a gamma probe," Surg. Oncol. 2(6), 335-339; discussion 340 (1993).

[2] Giuliano, A. E., Kirgan, D. M., Guenther, J. M., and Morton, D. L., "Lymphatic mapping and sentinel lymphadenectomy for breast cancer," Ann. Surg. 220(3), 391-398; discussion 398-401 (1992).

[3] Albertini, J. J., Lyman, G. H., Cox, C., Yeatman, T., Balducci, L., Ku, N., Shivers, S. et al., "Lymphatic mapping and sentinel node biopsy in the patient with breast cancer," JAMA 276(22), 1818-1822 (1996).

[4] Krag, D., Weaver, D., Ashikaga, T., Moffat, F., Klimberg, V. S., Shriver, C., Feldman, S. et al., "The sentinel node in breast cancer--a multicenter validation study,” N. Engl. J. Med. 339(14), 941-946 (1998).

[5] Kim, T., Giuliano, A. E., and Lyman, G. H., "Lymphatic mapping and sentinel lymph node biopsy in early-stage breast carcinoma: a metaanalysis," Cancer 106(1), 4-16 (2006).

[6] McMasters, K. M., Tuttle, T. M., Carlson, D. J., Brown, C. M., Noyes, R. D., Glaser, R. L., Vennekotter, D. J. et al., "Sentinel lymph node biopsy for breast cancer: a suitable alternative to routine axillary dissection in multi-institutional practice when optimal technique is used," J. Clin. Oncol. 18(13), 2560-2566 (2000).

[7] Swenson, K. K., Nissen, M. J., Ceronsky, C., Swenson, L., Lee, M. W., and Tuttle, T. M., "Comparison of side effects between sentinel lymph node and axillary lymph node dissection for breast cancer," Ann. Surg. Oncol. 9(8), 745-753 (2002).

[8] Purushotham, A. D., Upponi, S., Klevesath, M. B., Bobrow, L., Millar, K., Myles, J. P., and Duffy, S. W., “Morbidity after sentinel lymph node biopsy in primary breast cancer: results from a randomized controlled trial," J. Clin. Oncol. 23(19), 4312-4321 (2005).

[9] Krag, D. N., Anderson, S. J., Julian, T. B., Brown, A. M., Harlow, S. P., Ashikaga, T., Weaver, D. L. et al., "Technical outcomes of sentinel-lymph-node resection and conventional axillary-lymph-node dissection in patients with clinically node-negative breast cancer: results from the NSABP B-32 randomised phase III trial," Lancet Oncol. 8(10), 881-888 (2007).

[10] Zhang, H. F., Maslov, K., Stoica, G., and Wang, L.V., "Functional photoacoustic microscopy for high-resolution and noninvasive in vivo imaging." Nat. Biotechnol. 24(7), 848-851 (2006).

[11] Wang, X., Pang, Y., Ku, G., Xie, X., Stoica, G., and Wang, L. V., "Noninvasive laser-induced photoacoustic tomography for structural and functional in vivo imaging of the brain," Nat. Biotechnol. 21(7), 803-806 (2003).

[12] Song, K. H., Stein, E. W., Margenthaler, J. A., and Wang, L. V., "Noninvasive photoacoustic identification of sentinel lymph nodes containing methylene blue in vivo in a rat model," J. Biomed. Opt. 13(5), 054033 (2008).

[13] Song, K. H., Kim, C., Cobley, C. M., Xia, Y., and Wang, L. V., "Near-infrared gold nanocages as a new class of tracers for photoacoustic sentinel lymph node mapping on a rat model," Nano Lett. 9(1), 183-188 (2009).

[14] Song, K. H., Kim, C., Maslov, K., and Wang, L. V., "Noninvasive in vivo spectroscopic nanorod-contrast photoacoustic mapping of sentinel lymph nodes," Eur. J. Radiol. 70(2), 227-231 (2009).

[15] Pramanik, M., Song, K. H., Swierczewska, M., Green, D., Sitharaman, B., and Wang, L. V., "In vivo carbon nanotubeenhanced non-invasive photoacoustic mapping of the sentinel lymph node," Phys. Med. Biol 54(11), 3291-3301 (2009).

[16] Erpelding, T. N., Chulhong, C., Pramanik, M., Jankovic, J., Maslov, K., Guo, Z., Margenthaler, J. A., Pashley, M. D., and Wang, L. V., "Noninvasive photoacoustic and ultrasonic imaging of rat sentinel lymph nodes with a clinical ultrasound system," Radiol., Submitted for publication (2009).

[17] Dean, J., Gornstein, V., Burcher, M., and Jankovic, L., "Real-time photoacoustic data acquisition with Philips iU22 ultrasound scanner," Proc SPIE 6856(1), 685622-685611 (2008).

[18] Köstli K. P., Frenz, M., Bebie, H., and Weber, H. P., "Temporal backward projection of optoacoustic pressure transients using fourier transform methods," Phys. Med. Biol. 46(7), 1863-1872 (2001).

[19] American National Standards Institute. "American national standard for the safe use of lasers ANSI z136.1-2007," Laser Institute of America, Orlando, FL (2007). 